Japane Pychological Rereanth

1994. Vol 36. No. 3.113-120

Special lssue : Gestalt Perception I

Invited Paper

\title{
Conditions of visibility of actual paths
}

\author{
GAETANO KANIZSA \\ Department of Psychology, University of Trieste, via Università 7. Trieste, Italy
}

PETER KRUSE

Department of Psychology, University of Bremen, 33 Bibliotheksstr., 2800 Bremen, Germany

RICCARDO LUCCIO

Deparment of Psychology, University of Trieste, via Università 7, Trieste, Italy

MICHAEL STADLER

Department of Psychology. University of Bremen, 33 Bibliotheksstr., 2800 Bremen, Germany

\begin{abstract}
Two experiments demonstrate that when dots move along circular, partially overlapping paths, the observer succeeds in detecting the circular motion only when the average distance of each dot from the other dots of the same path (DS) is minor to the average distance of each dot from the dots of the other paths (DO). If DS is clearly minor to DO the relative motion emerges. When there is non-clear difference between DO and DS, no clear perceptual organisation emerges. The results are discussed in terms of theory of Prägnanz and of synergetics.
\end{abstract}

Key words: perception of movement, Prägnanz, synergetic.

On March 13, 1993 Gaetano Kanizsa spent all day at a meeting with me and other colleagues in Bologna. at our scientific publisher il Mulino. Going back to Trieste, on the train we discussed a quasi-final draft of the paper that follow's. We parted at 9.30 p.m. He looked ven healthy and was really happy. Another paper was almost finished. Three hours later I received the call: he had dead suddenly, without noticing what was happening. Together with Michael Stadler and Peter Kruse, I think that publishing this paper is perhaps the best way to remember him.

\section{Riccardo Luccio}

It is known that often the perceived movement of an object doesn't correspond to its physical motion. This is true for speed: Because it varies at the variation of the frame of reference inside which the movement occurs (Brown, 1931). It is true for direction also, as Ames' oscillating trapezoid (1955) and Johansson's precise analysis (1950) proved dramatically.

The description of what one sees looking at a rotating wheel is very simple and univocal: The wheel accomplishes a movement of linear translation; and meanwhile all its parts accomplish circular movements around the axis of the wheel itself. Indeed, only one point of the wheel, its centre, goes on a "physical" path that corresponds to the phenomenal path. All other parts accomplish motions that are different from what one sees. No physical path is circular. To see the actual motion one needs to isolate a single part from all other parts, as Rubin (1927) and Duncker (1929) first did. One can accomplish this in a very simple way fixating a little lamp or a phosphorescent dot somewhere on a wheel. Then, one lets the wheel roll in the dark along a plain. If the light is placed on the perimeter of the rolling wheel, one sees it running a path built up by a series of loops. 
This corresponds to the path physically followed by the lighted dot in the space. Mathematicians call this path a cycloid. In this case phenomenal path and physical path coincide. If one adds a second light to the periphery of the wheel, it isn't any more so easy to see the two cycloids: phenomenally a rotatory movement of each point around the other prevails (Cutting \& Proffitt, 1982). This phenomenal result stabilises itself and becomes coercive if we increase the number of the lighted points on the perimeter. Although it is still true that all the lighted points actually trace cycloids, we are quite unable to see them. We see on the contrary the points that rotate around an invisible centre and that displace themselves all together along another invisible plain. This phenomenal decomposition of the actual cycloid motion in a rotatory and a translatory component has been often considered a particularly convincing proof of the existence of a tendency to the Prägnanz in the perceptual system. Indeed. a circular movement is certainly "better" in the sense of regularity and fluidity, than a discontinuous and jerking cycloidal motion.

It is known that the postulation of a tendency to the prägnant result, which should be inherent to the visual system, is an assumption not only of the Gestalt psychology in its classic version, but widely granted in nongestaltist circles too. The validity of the assumption depends in our opinion on the meaning that one attributes to the term "Prägnanz". Kanizsa and Luccio (1986) have insisted that in gestalt tradition, Wertheimer as well as the other researchers used this term with two deeply different meanings. The first meaning refers to a phenomenal quality that some perceptual patterns have; we experience them as "better", more ordered, better accomplished, compared with others that are "bad", imperfect, disharmonic, ill accomplished. In this case "prägnant" is synonymous with singular (ausgezeichnet). In a second meaning, one uses the term "prägnant" to indicate a process that runs not at chance, but that is ruled by simplicity and economy principles, and that tends towards a state of dynamical balance, which has a maximum of stability and resistance to change. In this case, "prägnant" is equivalent to lawful.

The two meanings do not exclude each other, but they are not necessarily joined. A percept is always the result of a lawful process, but very seldom it is phenomenally singular. Though Wertheimer and other gestaltists apparently do not take in account such a distinction, when they insist that in the visual system a tendency to the Prägnanz exists, according to Kanizsa and Luccio to speak about such tendency is only meaningful if we bear this distinction firmly in mind. Kanizsa and Luccio deny that a tendency to the Prägnanz exists at all, when with it is understood as a tendency to singularity.

In their view, the behavior of the visual system is not characterised by a tendency to singularity, but by a tendency to stability. Though proximal stimulation undergoes a continuous process of transformation, our phenomenal world is usually a stable world, constituted by objects that preserve a high degree of constancy as to size, shape, colour, identity. The stability is the result of a capacity of self-organisation displayed by the visual system. This has already been claimed by Gestalt theory using arguments that today many students have revisited, updated and refined, also at the light of progresses made by neuroscience (Stadler \& Kruse, 1986; Hatfield \& Epstein, 1985; Haken \& Stadler, 1990). The system self-regulates according to principles that are essentially the ones that Wertheimer individuated (proximity, similarity, common fate, and so forth). The synergetic or conflicting action of such principles tends to a perceptual result that is better in 
the sense of maximal stability (i.e., less reversible, less ambiguous), and not to the better result in sense of the esthetically agreeable, prototypical, or singular. The most cases that are referred to in the literature as evidence of a tendency to singularity are, according to Kanizsa and Luccio, casual results of these organisational principles. The possibility of a phenomenally "singular" appearance is only a by-product. Singularity is not the "end" to which the self-organisational process tends. Kanizsa and Luccio support their claim with a series of counterexamples. In these counterexamples the conditions of a singular result exist, but does not appear. The phenomenal solution preferred by the visual system does not show characteristics like regularity, symmetry, prototypicity, which are the peculiarities of Prägnanz, if understood as singularity.

One counterexample can be demonstrated for the perceived shape of the path of a movement. If three dots move along three circular paths partially overlapped (see Figure 1), we don't succeed in seeing the actual paths. What we see is an elastic triangle rotating and twisting in space. Increasing the number of the dots moving on each path from one to five, the patterns are still invisible. In the area in which the circular paths overlap, the dots form continuously transforming and disrupting groups. The overall

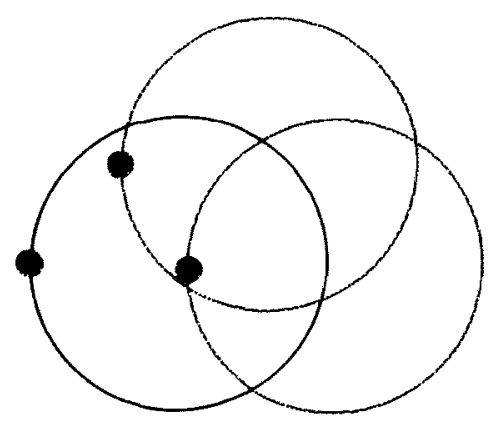

Figure 1. The dots move along the three circular, partially overlapping paths. impression is of great disorder.

The observer succeeds in detecting the circular motions only when there are more than six dots on each path. Obviously, there is a problem of relative distance between dots. Note that the observer is quite aware of the existence of the three distinct circular paths: His or her task is precisely to succeed in detecting them. The phenomenal impression is one of confusion, of brownian movement of dots upsurging from the middle of the configuration. This phenomenon was first seen by us informally; the following experiment was aimed to precise its conditions.

\section{Experiment I}

Apparatus. On the standard screen of a PET 2001 Commodore some dots appeared that were moving along three circular, partially overlapping paths (see Figure 1). The number of the dots was equal in the three paths, and there were eight conditions (15, $10,8,6,5,4,3,2$ dots for each path). The speed was 30 revolutions per minute; the ray of each path was $3 \mathrm{~cm}$; the centres of the paths were posed on the vertices of an equilateral triangle with the sides of $3 \mathrm{~cm}$ (like the ray). The size of the dots moving on the paths was 1 pixel. The positions of the dots were equidistant on each trajectory, and occupied corresponding positions on the three trajectories.

Subjects and procedure. Subjects were 16 students ( 9 females and 7 males) of the Faculty of Philosophy, the University of Trieste, participating in courses of Psychology on perception. The age ranged from 19 to 27 . At the beginning all subjects were told that they would see dots moving along circular paths on a screen. Their task was to detect the paths. First, they were shown the condition with 15 dots per path, in which the circular paths were highly evident; 
second, they were shown a condition with only one dot per path. In this case, although all subjects knew there were circular paths, no subjects could see them clearly; they saw instead the vertices of a virtual triangle, that moved circularly on the screen; it was possible to detect the paths only paying attention to a single dot each time, and keeping its track. At this point, the subjects were told that they will be shown several situations with a variable number of dots, always moving along circular paths; their task was to assess the clearness with which they could detect the paths, giving a rating on a 7 points" scale $(1=$ minimum of clearness; $7=\operatorname{maxi}$ mum). All the conditions were presented to all subjects in random order, with four presentations for each conditions.

Results

Results are very clear-cut. The subjects can detect highly confidently the paths, from 6 dots up, and are highly unable to do so from 4 dots down. The average ratings are 6.4 (10 dots), 6.2 (8 dots), 5 (6 dots), 4.8 (5 dots), 2.7 (4 dots), 1.9 (3 dots), 1.9 (2 dots) (see Figure 2).

An ANOVA for repeated measures was performed. There is a highly significant difference between conditions $\left(F_{0.105,}=151.053\right.$, $p<.00001)$. In the post hoc analysis the Scheffe $F$-test shows no difference between

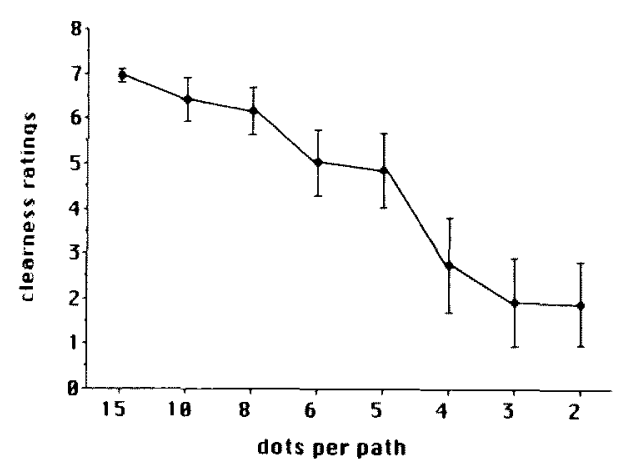

Figure 2. Average clearness ratings in function of the number of dots per path. conditions 15, 10,8; no difference between conditions 6 and 5; no difference between conditions 4, 3, and 2; but conditions 15,10 , and 8 differ from all the other conditions at $95 \%$; and conditions 6 and 5 differ from all the other conditions at $95 \%$. Thus, in a range from 8 to 15 dots the paths are clearly detectable; in a range from 2 to 4 dots it is very difficult to detect them. Conditions 5 and 6 are intermediate between such extremes.

From a qualitative point of view, we can say the following about the number of dots per path:

- one-two dots: subjects report that they see the vertices of one (respectively two) virtual triangle that rotates (rotate together) on the screen.

- three dots: the prevailing perceptual result is a sort of pulsation, with dots moving alternatively inward and outward, with reference to the centre of the figure.

- 4-5 dots: any regularity disappears: subjects see somewhat like a chaotic brownian motion in the centre of the figure; or dots springing up from the centre, in a process of continuous new generation. At the periphery of the figure the individual dots can trace fragments of circular paths, but these paths are completely lost towards the middle.

- 6-15 dots: the circular paths appear more and more clearly with the increasing of the number of dots.

Discussion

In a paper on minimum principle and perceived movement, Cutting and Proffitt (1982) stressed the importance at the distinction between absolute, common, and relative motion. It is very clear what absolute motion is mainly after the seminal work of Rubin (1927), Duncker (1929), and more recently Johansson (1950, 1973); all authors agree that the Konstanzannahme (a name created by Koffka), according to which elementist psychologists accounted perceived motion, 
has to be rejected. However, ideas are less clear about common and relative motion. The first is the apparent motion of the whole configuration relative to the observer, and the second is the apparent motion of each element relative to other configural ones.

In general, authors disagree concerning the weight to be attributed to common and relative motion. Some authors claim that observers perceive first common motion, and relative motion is left as residual. Some others claim that observers perceive first relative motion, and common motion is left as residual. Cutting and Proffitt, however, have shown that there are two simultaneous processes that correspond to common and relative motion. In both the minimum principle is involved. The prevalence of either is a matter of which process reaches first a minimum.

Results of our experiment can be analysed according to this view. In this case we have the same basic ambiguity in speaking about minimum principle that we have pointed out speaking about Prägnanz.

According to our results, in the situation with 1-2 dots there is a very clear prevalence of common motion: Subjects perceive the whole figure like one or two triangles, that move circularly all together. One does not see the individual circular paths, because the three dots that are the vertices of the triangles belong each to a different path, and one sees the triangles rotating as a whole.

With six or more dots, relative motion clearly prevails. Notice that in this case relative motion and absolute motion coincide. Here we see clearly the dots moving along circular paths. These dots, however, are seen relatively to the other dots, forming three independent rotating circumferences. Of course, coincidence between relative and absolute motion is very often in everyday perception. This is possibly the reason for the difficulty of disentangling the two kinds of motion in early studies.
According to minimum principle, one can consider the perceptual solutions for $1-2$ and 6-15 dots to be "good" solutions. But what can we say, about the 3-5 dots conditions? Here no "good" solution is seen, neither for common motion (3-5 triangles rotating together), nor for relative motion (rotating circumferences). Moreover, why is there this dramatical change in appearance of configurations, varying the number of dots?

Notice that in all conditions the dots (except, of course, when they are only 1 or 2 per path) occupy in each path the vertices of regular polygons inscribed in a circumference (equilater triangle, square, regular pentagon, and so on). Notice also that when the average distance of each dot from the other dots of the same path (DS) is different, according to the class of the polygon, the average distance of each dot from the dots of the other paths (DO) is always the same, leaving constant the relative position of the three paths. If the centres of the paths are on the vertices of an equilater triangle, as in our case, DO is always the same, with $3,4,5 \ldots$ 15 dots per path. (We have discovered this geometric regularity with some surprise a posteriori.)

Now, in the figures that we used in the experiment, DO was always $4.96 \mathrm{~cm}$. The DSs were respectively $3.96 \mathrm{~cm}$ ( 15 dots), $4.22 \mathrm{~cm}$ (10 dots), $4.27 \mathrm{~cm}$ (8 dots), $4.48 \mathrm{~cm}$ (6 dots), $4.65 \mathrm{~cm}$ ( 5 dots), $4.83 \mathrm{~cm}$ (4 dots), $5.20 \mathrm{~cm}$ ( 3 dots), $6 \mathrm{~cm}$ ( 2 dots). This suggested to us the hypothesis that the difference between DO and DS could be a crucial factor for the prevalence of either common or relative motion. When DO is clearly minor to DS common motion prevails. When DS is clearly minor to DO relative motion prevails. To test this hypothesis, a second experiment was performed, varying the distance between paths and leaving constant the number of dots per path. 


\section{Experiment II}

Apparatus and procedure. Conditions were very similar to the first experiment. In this case, the dots were presented through a Digital VC105, with display HP1310A. The number of the dots was always three per path. The speed was 30 revolutions for minute. The centres of the paths were on the vertices of an equilater triangle, varying for length of side with five conditions, according to different DOs: $7.48 \mathrm{~cm}$ (A), $8.22 \mathrm{~cm}$ (B), $8.66 \mathrm{~cm}$ (C), $9.41 \mathrm{~cm}$ (D), $9.94 \mathrm{~cm}$ (E). The DS was always 8.66 (equal to DO in $\mathrm{C}$ condition).

Subjects were 16 students ( 10 females and 6 males) of the University of Bremen, attending courses of Psychology. The age ranged from 19 to 27 years. The instructions were similar to those of the first experiment. To begin with, all subjects were told that they would see on the screen dots moving along circular paths; their task was to detect the paths. After this were presented two conditions which were not used later during the experiment: $\mathrm{DO}=13.01 \mathrm{~cm}$, in which the circular paths were highly evident; $\mathrm{DO}=$ $6.46 \mathrm{~cm}$, in which the circular paths were virtually undetectable. The subjects were told that their task would be to assess the clearness with which they could detect the paths, giving a rating on a 7 points scale $(1=$ minimum of clarity, as in DO $=6.46 ; 7=$ maximum, as in DO $=13.01$ ). The conditions were presented to all subjects in random order, with four presentations for each conditions.

Results

Again the results are very clear-cut. The average ratings are $3.5(\mathrm{~A}), 3.34(\mathrm{~B}), 4.06$ (C), 4.75 (D), 4.59 (E) (see Figure 3).

An ANOVA for repeated measures was performed. There is a highly significant difference between conditions $\left(F_{44,75)}=9.24, p<\right.$
.0001 ). The post hoc analysis shows at the Fisher's PLSD test a significant (.95) difference between condition $A$ and conditions $D$ and $\mathrm{E}$; between condition $\mathrm{B}$ and conditions $\mathrm{C}, \mathrm{D}$, and $\mathrm{E}$; between conditions $\mathrm{C}$ and $\mathrm{D}$.

From a qualitative point of view, subjects reported that in conditions $\mathrm{A}$ and $\mathrm{B}$ they saw the pulsation, with dots moving alternatively inward and outward, with reference to the centre of the figure, in conditions $\mathrm{D}$ and $\mathrm{E}$ the circular paths appeared clearly. The condition $\mathrm{C}$ oscillated between such appearances.

\section{Discussion}

The results of the second experiment support clearly the hypothesis. When DOs are minor than DS, it is very difficult to detect the circular paths. A perceptual organisation in which the dots are moving along these paths is possible only if the average distance between dots on a path is less than the average distance of each of them to the dots of the other paths. Thus, the proximity of dots proves itself to be a crucial factor in perceptual organisation of phenomenal motion.

This is no surprise. Several authors have stressed the importance of this factor in similar situations, for instance recently Ishiguchi (1991). Ullman $(1979,1984)$ has demonstrated how important proximity is in the perceptual solution of the so called "correspondence problem", which comes up when the

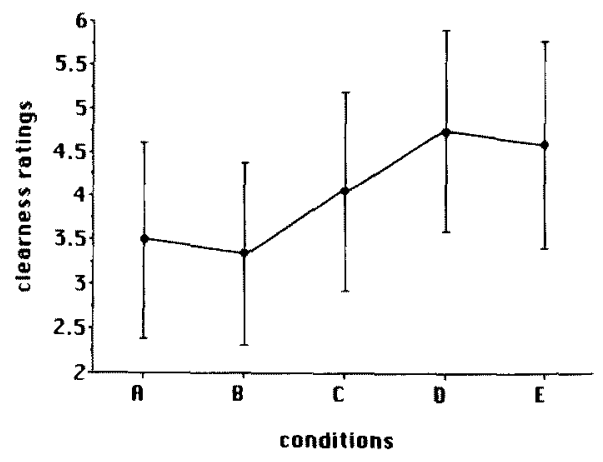

Figure 3. Average clearness ratings in function of the distance between paths. 
apparent moving objects are more than two and when therefore there is a plurivocity of perceptual outcomes (exactly, for $N$ objects. the possible solutions are $N$ ! - see figure 5) (see also Dawson and Pylyshyn, 1988, Marr. 1982, pp. 182ff.).

But there is a difference between the situation of the described experiments and the other perceptual situations previously investigated. The perceptual result that one gets, when no circular paths are seen, is far from being simple, regular, prägnant. Indeed, one can think that the condition to have such perceptual outcomes are present in the stimulus. The dots actually move along circular paths, and circles are highly prägnant figures. And when common motion prevails, as in conditions 1 and 2 of the first experiment, we have equilateral triangles moving together, again highly prägnant figures. Yet, in conditions A and $\mathrm{B}$, as in conditions 3-5 of the first experiment, neither circles nor triangles can be seen.

We can conclude that with increasing the number of dots in the first experiment the visual system undergoes a phase transition from common motion to relative motion passing an instable situation with no clear perceptual organisation. The relevant control parameter (in terms of "synergetics"; Haken \& Stadler, 1990) or the higher order variable (in terms of "ecological perception") is the difference between the average distance from the dots of other paths (DO) and the average distance from the dots of the same path (DS). If DO is clearly minor to DS the order parameter of common motion emerges and the system is in a stable attractor state. If DS is clearly minor to DO the order parameter of relative motion emerges and the system is in a totally different stable attractor state. In this theoretical view of the experimental data it can be predicted that one should be able to demonstrate a hysteresis effect by gradually approaching the phase transition from the sit- uation of stable common motion and form the situation of stable relative motion.

References

Ames, A., Jr. 1955 An interpretative manual. The nature of our perceptions, prehensions, and behavior. Princeton: Princeton University Press.

Brown, J. F. 1931 The visual perception of velocity. Psychologische Forschung, 14, 199-232.

Cutting, J. E., \& Proffitt, D. R. 1982 The minimum principle and the perception of absolute, common, and relative motion. Cognitive Psychology, 14, $211-246$

Dawson, M., \& Pylyshyn, Z. W. 1988 Natural constraints in apparent motion. In Z. W. Pylyshyn (Ed.), Computational processes in human vision. Norwood, NJ: Ablex. Pp. 99-120

Duncker, K. 1929 Über induzierte Bewegung Psychologische Forschung, 12, 180-259.

Goldmeier, E. 1982 The memory trace: Its formation and its fate. Hillsdale, NJ: Lawrence Erlbaum Associates.

Haken, H., \& Stadler, M. 1990 Synergetics of cognition. Berlin: Springer.

Hatfield, G., \& Epstein, W. 1985 The status of minimum principle in the theoretical analysis of visual perception. Psychological Bulletin, 97, 155176.

Ishiguchi, A. 1991 Effects of change of spatial distance between moving dots on their apparent linkage. Japanese Psychological Research, 33, 86-96

Johansson, G. 1950 Configurations in event perception. Uppsala: Almkvist Wilsell.

Johansson, G. 1973 Visual perception of biological motion and a model for its analysis. Perception \& Psychophysics, 14, 201-211.

Kanizsa, G., \& Luccio R. 1986 Die Doppeldeutigkeiten der Prägnanz. Gestalt Theory, 8, 99-135.

Köhler, W. 1920 Die physischen Gestalten in Ruhe und im stationären Zustand. Braunschweig: Vieweg.

Mar, D. 1982 Vision. San Francisco, CA: Freeman.

Rausch, E. 1966 Das Eigenschaftsproblem in der Gestaltheorie der Wahrnehmung. In W. Metzger \& H. Erke (Hsg.), Handbuch der Psychologie. Bd 1/1. Wahrnehmung und Bewußstein. Göttingen: Hogrefe.

Rubin, E. 1927 Visuell wahrgenommene wirkliche Bewegungen. Zeitschrift für Psychologie, 103, 384-392. 
Stadler, M. \& Kruse, P. 1986 Gestaltheorie und Theorie der Selbstorganisation. Gestalt Theory, 8. 75-98.

Ulman. S. 1979 The interpretation of visual motion. Cambridge, MA: MIT Press.
Ullman, S. 1984 Maximizing rigidity: The incremental recovery of 3-D structure from rigid and non rigid motion. Perception, 13, 255-274.

(Received Dec. 10, 1993; accepted May 7, 1994) 